\title{
Adenovirus co-expressing CD40 ligand and interleukin (IL)-2 contributes to maturation of dendritic cells and production of IL-12
}

\author{
ZHI GUO, HONG-YAN GAO, TIAN-YANG ZHANG，JIN-XING LOU，KAI YANG， \\ XIAO-DONG LIU, XUE-PENG HE and HUI-REN CHEN \\ Department of Hematology, Beijing Military General Hospital, Beijing 100700, P.R. China
}

Received October 11, 2015; Accepted September 14, 2016

DOI: $10.3892 /$ br.2016.773

\begin{abstract}
The aim of the present study was to construct a chimeric adenovirus (Ad)5/F35 co-expressing human CD4O ligand (CD4OL) and interleukin (IL)-2 (Ad5/F35 CD40L-IL-2). The infection efficiency to human monocyte-derived dendritic cells (Mo-DCs), expression of genes, phenotype changes and IL-12 production of Mo-DC by Ad5/F35 CD40L-IL-2 were investigated. CD40L and IL-2 from total RNA extracted from human peripheral blood mononuclear cells (PBMCs) were cloned by reverse transcription-polymerase chain reaction and used to construct Ad5/F35 CD40L-IL-2. The infection efficiency, expression of CD40L, and phenotype changes of Mo-DC infected with Ad5/F35 CD40L-IL-2 were analyzed using flow cytometry. The quantities of IL-2 and IL-12 in the supernatants of Mo-DC following infection of Ad5/F35 CD40L-IL-2 were measured by enzyme-linked immunosorbent assay. The CD40L and IL-2 genes were successfully cloned and the Ad5/F35 CD40L-IL-2 was constructed. Ad5/F35 CD40L-IL-2 efficiently infected Mo-DCs with an infection efficiency of $>75 \%$, and the infected Mo-DCs expressed CD40L and secreted IL-2. The expression levels of cluster of differentiation (CD)80, CD86, CD40, and human leukocyte antigen-antigen D related on Mo-DC were moderate; however, CD83 was low prior to infection of Ad5/ F35 CD40L-IL-2. Those molecules, particularly CD83, were markedly upregulated $24 \mathrm{~h}$ after the infection. Increasing quantities of IL-12 in the supernatants were detected subsequent to infection at different time points in a time-dependent manner. Thus, Ad5/F35 CD40L-IL-2 efficiently infected human Mo-DCs and its products, CD40L and IL-2, were subsequently expressed. In addition, infection with Ad5/F35
\end{abstract}

Correspondence to: Dr Zhi Guo, Department of Hematology, Beijing Military General Hospital, Building 3, 5 Nanmencang Dongsishitiao, Beijing 100700, P.R. China

E-mail: guozhi02@163.com

Key words: CD40 ligand, interleukin-2, interleukin-12, chimeric adenovirus 5/F35, dendritic cells
CD40L-IL-2 stimulated the maturation of Mo-DC and high levels of IL-12 production.

\section{Introduction}

The dendritic cell (DC) tumor vaccine is currently one of the most common active immunity strategies for tumor treatment. The use of DCs in vitro and following antigen stimulation, as a therapeutic cancer vaccine induced specific immune responses and the corresponding tumor antigens have been investigated in a large number of animal experiments and confirmed in vitro, as well as in numerous clinical trials (1-3). Culture of DCs in vitro is achieved with DC precursor cells, such as cluster of differentiation (CD) $14^{+}$cells, that differentiate into immature DCs via stimulation with granulocyte-macrophage colony-stimulating factor (GM-CSF) and interleukin-4 (IL-4). The preparation of DC as a specific tumor vaccine is completed upon antigen stimulation and DC maturation. The induction of DC maturation is particularly important and an active tumor immune response may only be produced in mature DCs (4), with immature DCs leading to immune tolerance (5). Currently the most common method for inducing DC maturation in the clinical setting is incubation for $24-48 \mathrm{~h}$ with a variety of cytokines, including IL-1, IL-6, tumor necrosis factor (TNF)- $\alpha$ and prostaglandin E2 (PGE2) in immature DCs (6); however, this method is time-consuming and costly. In addition, the gene transduction method is often adopted to modify DCs to enhance their ability to stimulate the production of tumor-specific cytotoxic T lymphocytes (CTLs). Furthermore, one of the most commonly used methods is infecting DCs with adenovirus vectors to express various genes (7). The current study established a chimeric adenovirus (Ad) 5/F35 mosaic type adenoviral vector co-expressing CD40 ligand (CD40L) and IL-2, which was able to efficiently infect human monocyte-derived DCs (Mo-DC) and induced the maturation of Mo-DC and IL-12.

\section{Materials and methods}

Reagents and materials. EcoRI, BamHI, NcoI and XhoI double-enzyme were purchased from Takara Bio, Inc. (Otsu, Japan), husion ${ }^{\circledR}$ High-Fidelity DNA Polymerase and T4 DNA 
ligase were purchased from New England BioLabs, Inc. (Ipswich, MA, USA), and a ThermoScript ${ }^{\mathrm{TM}}$ kit, liposome Lipofectamine $2000^{\mathrm{TM}}$ and the eukaryotic expression vectors, pcDNA3.1/myc-His(-)B were purchased from Invitrogen (Thermo Fisher Scientific, Inc., Waltham, MA, USA). The antibodies for flow cytometry (CD80-PE (cat. no. 560925; clonality, L307.4; mouse, mouse IgG1; dilution, 1:20), CD86-PE (cat. no. 550616; clonality, MOPC-31C; mouse; mouse IgG1; dilution, 1:20), CD83-PE (cat. no. 557720; clonality, R3-34; mouse; mouse IgG1; dilution, 1:20), CD40-PE (cat. no. 560963; clonality, 5C3; mouse, mouse IgG1; dilution, 1:20), CD40L-PE (cat. no. 552559; clonality, hCD40L-M90.1; mouse, mouse IgG1; dilution, 1:20), and human leukocyte antigen-antigen D related (HLA-DR)-PE (cat. no. 562804; clonality, G46-6; mouse, mouse IgG1; dilution, 1:20)) were purchased from BD Pharmingen (San Diego, CA, USA), and the RNA extraction kit (RNeasy Mini kit) was purchased from Qiagen, Inc., (Valencia, CA, USA). Plasmid extraction, purification and gel extraction kits were purchased from Omega Bio-Tek, Inc. (Norcross, GA, USA) and The Cre-lox system recombinant adenovirus systems were purchased from Microbix Biosystems Inc. (Mississauga, ON, Canada). The IL-2 and IL-12 enzyme-linked immunosorbent assay (ELISA) kits were purchased from Diaclone SAS (Besançon, France) and all the culture mediums and fetal bovine serum (FBS) were purchased from Gibco (Thermo Fisher Scientific, Inc.). Lymphocyte Separation Medium was purchased from Haoyang Biological Technology Co., Ltd. (Tianjin, China).

Cloning of human (h)CD40L and IL-2. Total RNA was extracted using RNA extraction kits following isolation of peripheral blood mononuclear cells (PMBCs) with Lymphocyte Separation Medium, according to the manufacturer's instructions. The first strand cDNA was synthesized according to the instructions of the ThermoScript ${ }^{\mathrm{TM}}$ kit. According to the GenBank registration accession numbers of CD40L and hIL-2 (NM_000074 and NM_000586, respectively), two pairs of primers were designed during the present study as follows: Upstream, 5'-gga att cgc cat ggt cga aac ata caa cc-3' (the EcoRI site was drawn from the 5'point, and the restriction sites are underlined) and downstream, 5'-cgg gat cet cag agt ttg agt aag cc-3' (the BamHI site was drawn from the 5'point) for CD40L; upstream, 5'-cgg gat cca tgg aca gga tgc aac tc-3' (the BamHI site was drawn from the 5'point) and downstream, 5'-ccc aag ctt caa gtc agt gtt gag atg atg-3' (the HindIII site was drawn from the 5'point) for IL-2. The amplified fragments were inserted into the restriction enzyme sites of the pcDNA3.1/myc-His(-)B eukaryotic expression carrier, and the pcDNA3.1(-) CD40 and pcDNA3.1(-) IL-2 were constructed separately to demonstrate the success of the cloned sequence.

Construction and amplification of the Ad5/F35 CD4OL-IL-2 adenovirus vector. In order to construct the co-expression adenovirus vector of CD40L and IL-2, construction of pDC315 CD40L-IL-2, the co-expressing adenovirus vector carrier of CD40L and IL-2-shuttle plasmid, was required. The CD40L fragment was sliced from the pcDNA3.1(-)CD40L, using the EcoRI and BamHI double-enzyme, and inserted into the corresponding restriction sites of the Adenovirus Shuttle Plasmid, pDC315 (Microbix Biosystems Inc.) to constitute the pDC315 CD40L. A pair of IL-2 primers was designed for the construction of IL-2, which is connected with the internal nucleic acid access site (internal ribosome entry site; IRES). Upstream, $5 \varnothing$-cat gcc atg gac agg atg caa ctc c-3 $\phi$ (the NcoI site was drawn from the $5 \notin$ point) and downstream, $5 \phi$-ccg ctc gag tca agt cag tgt tga gat-3ф (the XhoI site was drawn from the $5 \varnothing$ point). The IL-2 fragment was amplified by polymerase chain reaction (PCR) from the pcDNA3.1(-) IL-2 plasmid using NcoI and XhoI double-enzyme, and the NcoI and XhoI double-enzyme was also used in the pmRNA IRES-luc vector (8). The fragment of IRES pmRNA was recovered by gel electrophoresis, expression of CD40L and phenotype changes of Mo-DCs infected with Ad5/F35 CD40L-IL-2 were analyzed using flow cytometry, and pmRNA IRES-IL-2 was directly constructed from the connection of IL-2 and pmRNA IRES. The IRES-IL-2 fragment was recovered by gel electrophoresis (140 V for $20 \mathrm{~min}$ ) using the BamHI and XhoI double-enzyme and inserted into the corresponding sites of pDC515 GM-CSF to form pDC315 CD40L-IRES-IL-2 (abbreviated as pDC315 CD40L- IL-2).

The packaging of the Ad5/F35 CD40L-IL-2 adenovirus was performed by Beijing Benyuan Zhengyang Gene Technology Co., Ltd. (Beijing, China) and, subsequently, the virus seed was obtained and identified by PCR (using pDC315 (Beijing Benyuan Zhengyang Gene Technology Co., Ltd.) murine cytomegalovirus, Simian virus $40(1 \mu \mathrm{g} / \mu \mathrm{l} ; 10 \mu \mathrm{l})$ and a LabCycler thermocycler under the following conditions: $94^{\circ} \mathrm{C}$ for $30 \mathrm{sec}$, followed by annealing $\left(64^{\circ} \mathrm{C}\right.$ for $\left.30 \mathrm{sec}\right)$ and extension $\left(72^{\circ} \mathrm{C}\right.$ for $30 \mathrm{sec}$ ) for 30 cycles). The 293 cells infected with the virus seed were then amplified by cell culture (at $37^{\circ} \mathrm{C}$ for 72 h). Following freeze-thaw lysis of the 293 collected cells, the purified Ad GM-CSF-IL-2 was acquired through $\mathrm{CsCl}$ gradient centrifugation, which was performed twice. The TCID50 method to quantify IL-2 and IL-12 in the supernatants of Mo-DCs following infection of Ad5/F35 CD40L-IL-2 was used to identify the virus titer.

Culture of human monocyte-derived DC. Peripheral blood samples $(20 \mathrm{ml})$ were obtained from a healthy donors (one male, aged 36-years-old; written informed consent was obtained and the study was ethically approved by the review board of the Beijing Military General Hospital) and human PBMCs were isolated using lymphocyte separation medium and density gradient centrifugation. The adhesion method was adopted to culture the DCs, according to the method described in a previous study (9). Briefly, PBMCs were suspended with RPMI-1640 complete medium (RPMI-1640 medium with $10 \%$ FBS) and added to a $175-\mathrm{cm}^{2}$ disposable plastic culture flask $\left(2 \times 10^{6} / \mathrm{ml}\right)$. The non-adherent cells and medium were extracted after $1 \mathrm{~h}$, and $20 \mathrm{ml}$ complete medium containing RPMI-1640 supplemented with $800 \mathrm{U} / \mathrm{ml} \mathrm{GM-CSF}$ and $250 \mathrm{U} / \mathrm{ml} \mathrm{IL-4} \mathrm{was}$ added. After three days of culture at $37^{\circ} \mathrm{C}$, the above $20 \mathrm{ml}$ GM-CSF and IL-4 medium was supplemented. After a further five days of culture at $37^{\circ} \mathrm{C}$, phenotypic identification or detection for adenovirus infection were conducted.

DC infection with adenovirus vectors. DCs were cultured at $37^{\circ} \mathrm{C}$ for five days, and the cells were suspended with RPMI-1640 complete medium containing GM-CSF and IL-4, and collected by centrifugation $\left(100 \mathrm{xg}\right.$ at $37^{\circ} \mathrm{C}$ for $\left.5 \mathrm{~min}\right)$. The cell number 
was adjusted to $5 \times 10^{5}$ cells $/ \mathrm{ml}$ and added to a 6 -well plate ( $3 \mathrm{ml} /$ well). The Ad5/F35 CD40L-IL-2 or Ad5/F35 EGFP (10) was added (viral titer, 100 plaque-forming unit (pfu)/cell), and $2 \mathrm{~h}$ after infection, the cells were centrifuged $\left(100 \mathrm{x} \mathrm{g}\right.$ at $37^{\circ} \mathrm{C}$ for $5 \mathrm{~min}$ ) and collected. The cells were placed into the 6 -well plate following suspension with RPMI-1640 complete medium containing GM-CSF and IL-4. The supernatant was collected and frozen at $-20^{\circ} \mathrm{C}$ until subsequent ELISA testing, after 6,12 and $24 \mathrm{~h}$, and phenotype testing was performed when the DCs were collected after $24 \mathrm{~h}$. Eight DCs were infected with Ad5/ F35 enhanced green fluorescent protein (EGFP) and fixed with $4 \%$ polyformaldehyde. The cells were analyzed under a fluorescence microscope to observe GFP expression.

Flow cytometric analysis. Flow cytometry was performed to identify DC phenotypes and assess the infection efficiency of the adenovirus vector in the DCs. The cells were collected, centrifuged (100 x g for $5 \mathrm{~min}$ ) and washed with phosphate-buffered saline (PBS) once. Following an additional centrifugation (100 x g for $5 \mathrm{~min}$ ), if adenovirus infection efficiency was to be detected, this was analyzed following resuspension with $400 \mu \mathrm{l}$ PBS. However, for phenotypic identification, a monoclonal antibody was added to the cells and incubated for $30 \mathrm{~min}$ in the dark at room temperature, and then washed two times with PBS. Subsequent to resuspension, the cells were exposed to Lm400 PBS, and flow cytometry was performed using a Beckman Coulter four-color flow cytometer (Coulter Epics XL; Beckman Coulter, Inc., Brea, CA, USA).

ELISA detection. After thawing of the culture medium, which was diluted five times, it was collected at each time-point $(6,12$ and $24 \mathrm{~h})$, and the level of IL-2 and IL-12 protein in the supernatant was detected according to the instructions of the ELISA kit.

Statistical analysis. Continuous variables are presented as means \pm standard deviation and $95 \%$ confidence interval for the descriptive statistics. Differences between the two groups were assessed by Student's t-test for continuous variables. For comparison of percentages (gender, multiorgan dysfunction syndrome, antibiotic substance classes and mortality) the $\chi^{2}$ test was employed using SPSS 13.0 statistical software (SPSS, Inc. Chicago, IL, USA). P $<0.05$ was considered to indicate a statistically significant difference.

\section{Results}

Cloning of the CD4OL and IL-2 genes. Total RNA that was obtained from healthy human PBMCs was synthesized to the first strand cDNA by reverse transcription. Two fragments of 700 and 450 bp size, were obtained by PCR amplification of the CD40L and IL-2 primers, and was inserted to the corresponding locus of the eukaryotic expression vector, pcDNA3.1/myc-His (-) B after a restriction enzyme fragment, then identified by the corresponding primers. The positive clones were sequenced by Shenggong (Beijing) Biological Technology Co., Ltd. (Beijing, China), and it was confirmed that the sequence of the CD40L and IL-2 clones were the same as CD40L (serial number, NM_000074; 708 bp) and IL-2 (serial number, NM_000586 (462 bp) according to GenBank.

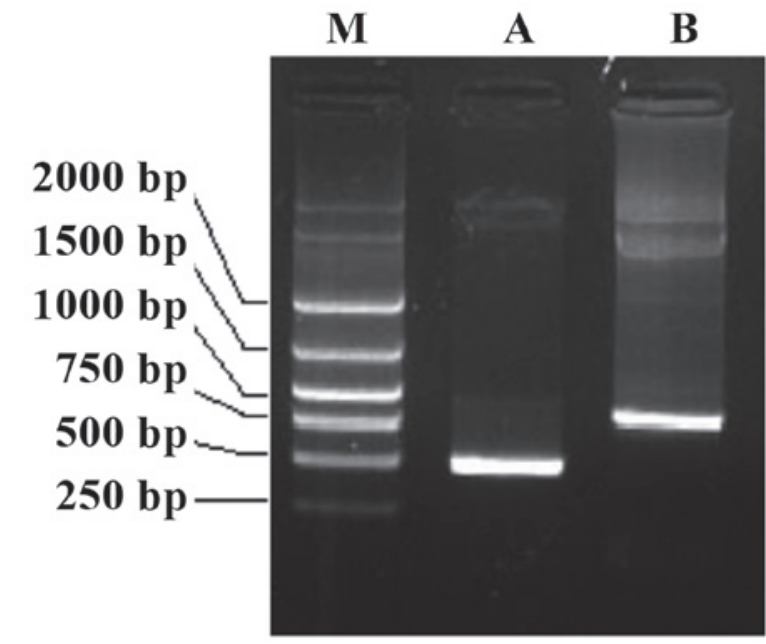

Figure 1. DNA results with IL-2 and CD40L primers following polymerase chain reaction amplification of the Ad5/F35 CD40L-IL-2 virus. Lane A, IL-2; lane B, CD40L. M, marker; CD40L, CD40 ligand; IL, interleukin.

Construction of Ad5/F35 CD40L-IL-2. CD40L was subcloned into an adenoviral shuttle plasmid, pDC315, and then the IRES-IL-2 fragment was inserted into the GM-CSF, so that the CD40L and IL-2 were connected in series through the IRES sequence, and under the regulation and promoter of the same cytomegalovirus (CMV). The constructed adenoviral shuttle plasmid, pDC315 CD40L-IL-2 was packaged to produce the virus within adenovirus packaging, and the virus DNA was extracted for subsequent identification. PCR amplification was performed using clone primers of CD40L and IL-2, and two fragments of the same size as the CD40L and IL-2 gene fragments were obtained (Fig. 1). This demonstrated that CD40L and IL-2 were in the DNA adenovirus genome. Furthermore, $10^{10}-10^{11} \mathrm{pfu}$ viral titers were obtained by repeated amplification of virus seeds in 293 cells and purification by $\mathrm{CsCl}$ gradient centrifugation.

Phenotypic characteristics of Mo-DCs. DCs were cultured for five days and stained with CD86, CD80, CD83, CD40 and HLA-DR antibodies. The results indicated that following five days of culturing, DCs demonstrated the immature phenotype, with moderate expression ( $>50 \%$ ) of co-stimulatory molecules, CD80, CD86 and CD40 and major histocompatibility complex (MHC) II molecule, HLA-DR and low expression $(<20 \%)$ of CD83 (Fig. 2).

Infection efficiency of Ad5/F35 adenovirus vector in Mo-DCs. The expression efficiency of EGFP was detected by flow cytometry after $24 \mathrm{~h}$ of infection of Mo-DC by Ad5/F35 EGFP or Ad5/F35 CD40L-IL-2 (virus titer, 100 pfu/cells), and the results indicate that the infection efficiency of Ad5/F35 EGFP to Mo-DC was 76.8\%. The expression of CD40L was detected by flow cytometry and the expression rate was identified to be $75.2 \%$, which was similar to EGFP (Fig. 3A). The green fluorescence was observed under a fluorescence microscope (Fig. 3B).

Maturation of Mo-DC by induction of Ad5/F35 CD40L-IL-2 and production of $I L-12$. The phenotypic changes of Mo-DC 


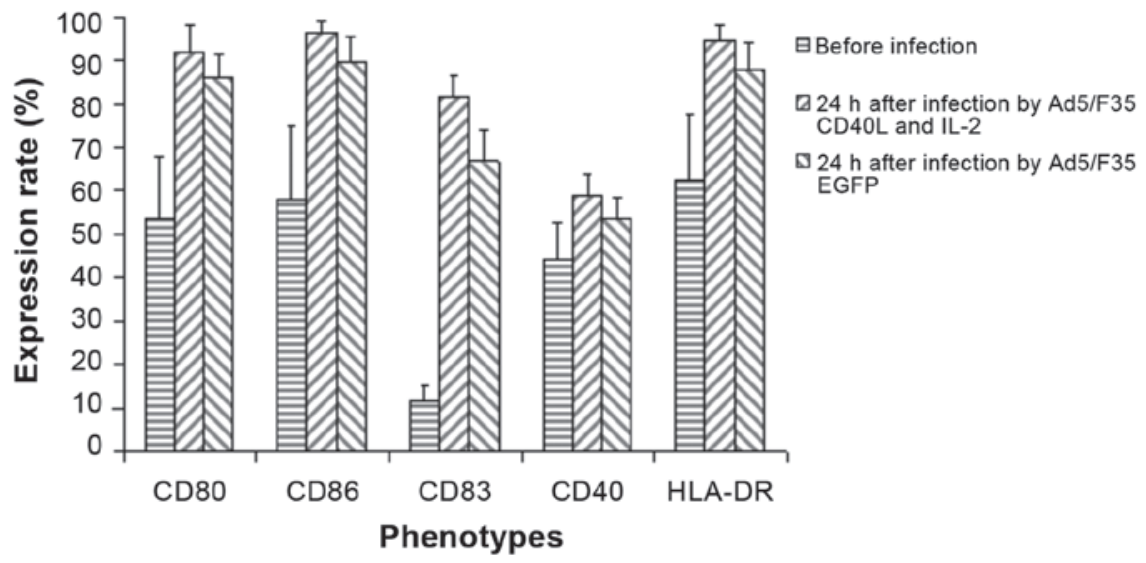

Figure 2. Phenotypic changes before and after adenovirus infection of monocyte-derived dendritic cells. CD, cluster of differentiation; HLA-DR, human leukocyte antigen-antigen D related; CD40L, CD40 ligand; IL, interleukin; EGFP, enhanced green fluorescent protein. P $<0.05$ vs. before injection.
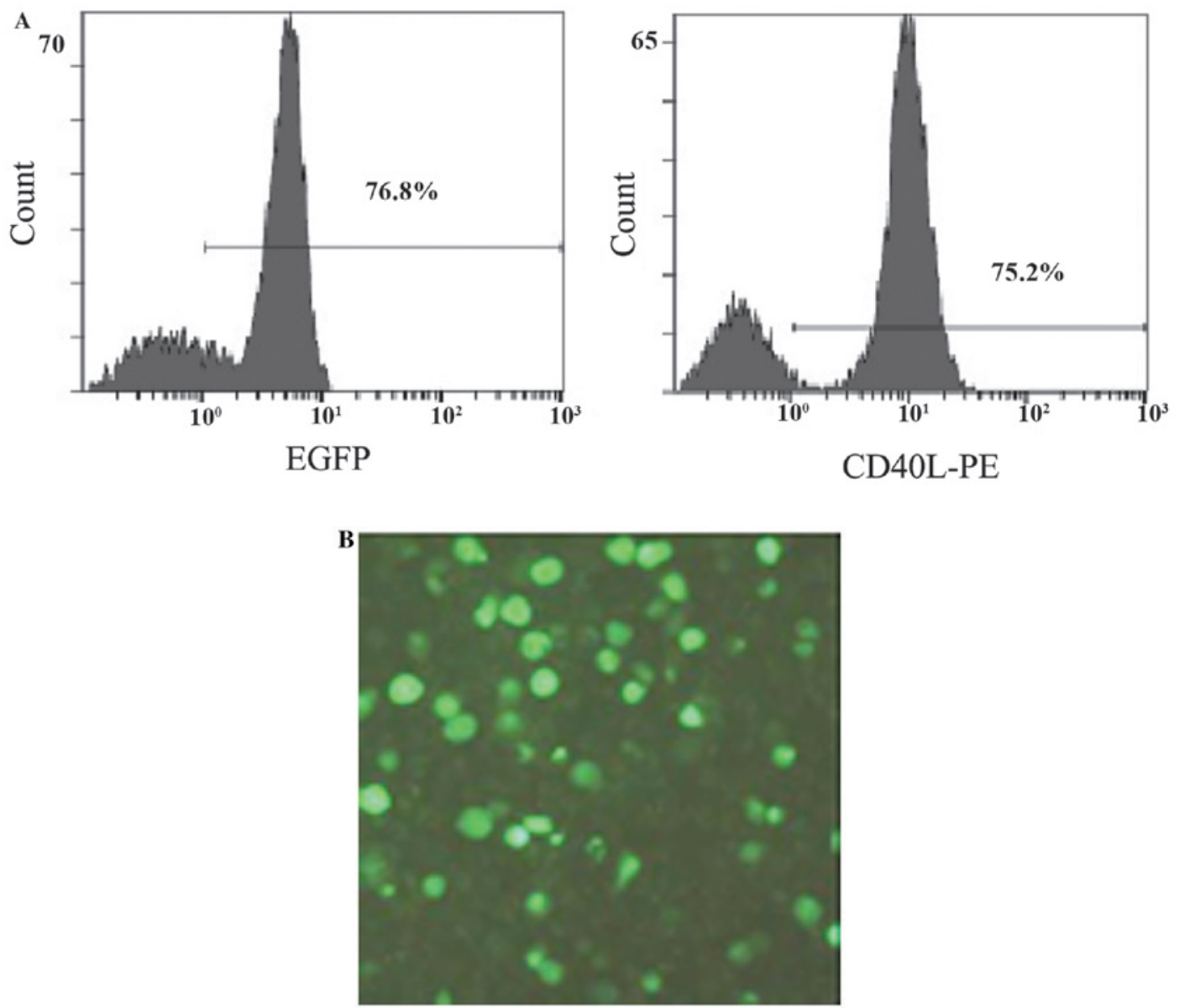

Figure 3. (A) Infection efficiency of Mo-DCs infected with Ad5/F35 adenovirus vector. (B) EGFP expression of Mo-DCs infected with Ad5/F35 EGFP after $24 \mathrm{~h}$ (magnification, x10). Mo-DC, monocyte-derived dendritic cell; EGFP, enhanced green fluorescent protein; CD40L, CD40 ligand.

were detected through flow cytometry $24 \mathrm{~h}$ after infection by Ad5/F35 EGFP or Ad5/F35 CD40L-IL-2 (virus titer, $100 \mathrm{pfu} / \mathrm{cells}$ ), and the results indicated that the expression of CD86, CD80, CD83, CD40 and HLA-DR was increased; however, after $24 \mathrm{~h}$, when DCs were infected CD40L-IL-2, the expression levels of CD86, CD80, CD83, CD40 and HLA-DR were increased more markedly (Fig. 3). This indicated that activation and maturation of DCs may be induced by autoinfection of Mo-DC, although phenotypic changes were more obvious when CD40L was expressed by DCs and DCs were trending towards a mature phenotype. ELISA was used to detect the contents of IL-2 and IL-12 in the cell supernatant at different time-points $(6,12$ and $24 \mathrm{~h}$ ) subsequent to Mo-DC infection with Ad5/F35 CD40L-IL-2 or Ad5/F35 EGFP (Fig. 4A). The expression of IL-12 was detected $6 \mathrm{~h}$ after Mo-DCs were infected with CD40L-IL-2 and the expression continued to 


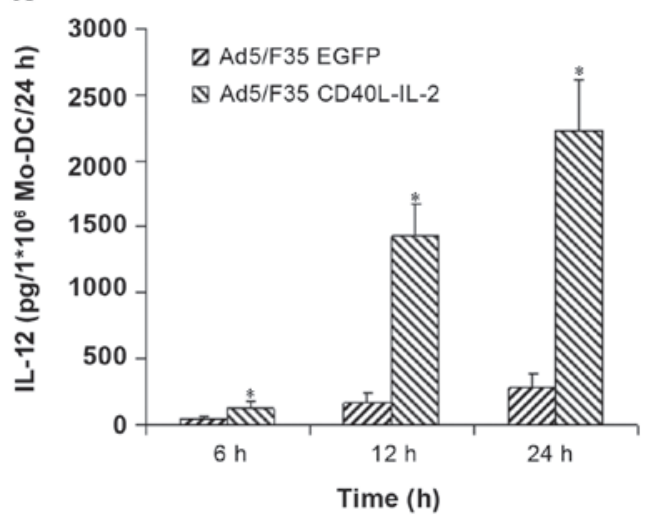

B

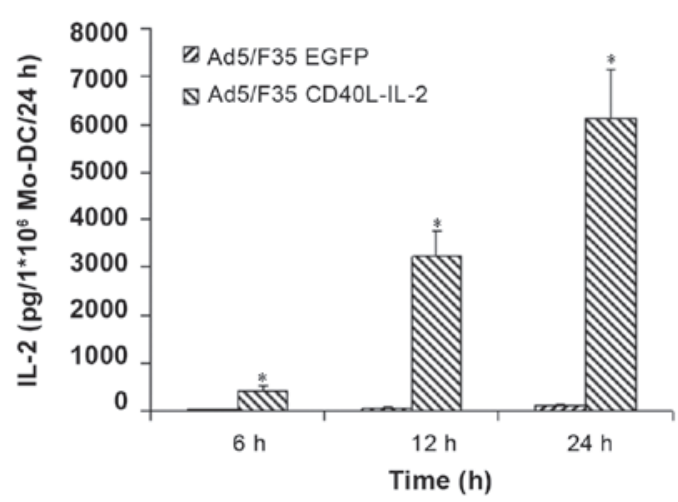

Figure 4. Expression levels of (A) IL-12 and (B) IL-2 on Mo-DCs infected with Ad5/F35 EGFP or Ad5/F35 CD40L-IL-2. IL, interleukin; Mo-DC, monocyte-derived dendritic cell; EGFP, enhanced green fluorescent protein; CD40L, CD40 ligand. "P<0.05 vs. Ad5/F35 EGFP.

rise after $24 \mathrm{~h}$. Although production of IL-12 was induced by infection with pure Ad5/F35 EGFP and exhibited a similar mode of secretion, the IL-12 secretion was significantly lower $(\mathrm{P}<0.05)$ when compared with the secretion from Mo-DCs after infection with Ad5/F35 CD40L-IL-2; the difference between the two was 2-8 times. In addition, following infection of Mo-DCs with Ad5/F35 CD40L-IL-2, high levels of IL-2 were detected in the culture supernatant; however, Mo-DCs infected with Ad5/F35 EGFP only exhibited low levels of expression (Fig. 4B).

\section{Discussion}

Tumor immunotherapy based on DCs is currently a popular tumor immunotherapeutic strategy. Subsequent to antigen stimulation by the tumor, and after being stimulated by tumor associated antigens, DCs process the antigens and deliver them to the $\mathrm{T}$ cells, and the initial (naïve) $\mathrm{T}$ lymphocytes differentiate into CTLs, which recognize and kill the corresponding tumor cells. CTL production by DCs require at least three signals (11): Tumor antigens, in the form of MHC-antigen peptide complex (the first signal), processed and delivered by $\mathrm{DCs}$, which stimulate $\mathrm{T}$ cells, including $\mathrm{CD}^{+}$and $\mathrm{CD}^{+}$ $\mathrm{T}$ lymphocytes. Co-stimulatory molecules of DC surface expression, such as CD80, CD86 and CD40 are the second signal and interact with co-stimulatory molecule ligand ions of $\mathrm{T}$ cell surface expression. The activated T cells, DCs and $\mathrm{CD}^{+} \mathrm{T}$ cells then secrete various cytokines (the third signal), including Th1 cytokines, such as IL-2, IL-12 and TNF-PGE2 that promote significant cell proliferation of responsive CD8+ T-lymphocytes, and thereby CTLs with tumor antigen specificity are produced. DC culturing in vitro is induced and differentiated mostly from the peripheral blood monocytes, CD14+ by GM-CSF and IL-4; however, DCs induced by these two types of cytokine are of the immature phenotype, which express low levels of co-stimulatory and MHC II molecules. Furthermore, these immature DCs promote organisms to produce specific antitumor immunity reactions as a tumor vaccine to stimulate the body to produce specific antitumor immune response. Bacterial lipopolysaccharides (LPSs) have been used to induce maturation of DCs in in vitro studies and animal models (12). In a previous study, DCs were incubated with a variety of cytokines, including IL-1, IL-6, TNF-PGE2 for 24-48 $\mathrm{h}$ (3); however, this method is time-consuming and costly. The interaction of CD40 and CD40L is known to be particularly important; the functions of DCs in vivo and the interaction of CD40L with the expression of $\mathrm{CD}^{+}$and CD40 on the surface of DCs is the condition required to produce tumor specific CTL with DC stimulation (13). Previous studies found that an effective specific antitumor immune response is produced by DCs independent of $\mathrm{CD}^{+}{ }^{+}$with the stimulation of anti-CD40 monoclonal antibody or CD40L $(14,15)$. DCs infected by adenovirus carriers $(16,17)$ and expressing CD40L effectively induced an antitumor immune response in animal experiments, and the induction of the antitumor immune response was associated with DC maturation induced by CD40L and the production of various cytokines $(18,19)$, particularly IL-12 (20). Certain DCs, which are of the mature phenotype, but cannot produce IL-12, are occasionally referred to as half-mature DCs, while only DCs of the mature phenotype and producing IL-12 are termed mature DCs. Only the mature DCs effectively induce an antitumor immune response (21).

The present study hypothesized that infecting DCs derived from PBMCs with a chimeric adenovirus 5/F35 carrier co-expressing CD40L and IL-2 induces mature phenotype DCs, producing large quantities of IL-12. The present study has the following characteristics: First, the co-expression of CD40L and IL-2 was demonstrated to enhance the antitumor effect. Consistent with this, CD40L expressed alone with an adenovirus vector has been demonstrated to effectively induce an antitumor immune response (22); however, marked proliferation of CTLs requires the stimulation of a variety of lymphocyte proliferation factors following production of tumor specific CTLs by DC stimulation, particularly IL-2. However, DCs and $\mathrm{CD}^{+}{ }^{+} \mathrm{T}$ lymphocytes also produce certain quantities of IL-2, although exogenous expression of IL-2 exerts more stable and durable functions. A previous study showed that CD40L and IL-2 exert a congenerous antitumor effect (23), and Rousseau et al applied these results from the in vitro and animal experiments into a clinical setting in which a mixture of primary cultured skin fibroblasts infected with Ad IL-2 and 
Ad CD40L, and autologous leukemia cells were administered by intradermal injection. The pertinent leukemia cell responses of the patients were then monitored (24). CD40L and IL-2 are co-expressed in the same carrier, and in future applications it may be possible to reduce the number of steps (by only producing a carrier) and reduce the cost. In addition, the maturation time of DCs in vitro may be significantly shortened following the infection of DCs with the adenovirus vector, as the process of DC maturation induced by CD40L was constant and did not change when DCs were infected by adenovirus in vivo or in vitro. Therefore, DCs may be inoculated following adenovirus infection, and the maturation process of DCs may be completed in vivo, rather than culturing for 24-48 $\mathrm{h}$ in vitro, as is necessary when inducing DC maturation with cytokines.

In addition, hematopoietic system cells, including DCs, may be used as carriers of chimeric adenovirus 5/F35, as it is possible to efficiently infect these cells. It was known that adenovirus type 5 from the hematopoietic system, including dendritic cells, have a low infection efficiency, as the cells of hematopoietic origin exhibit low Coxsackie adenovirus receptor (CAR) expression or a complete lack of CAR (25), and the infection of adenovirus type 5 into cells is dependent on the receptor. The mechanism of the chimeric adenovirus $5 / \mathrm{F} 35$ vector entering into cells involves passage through CD46, and the molecule is commonly expressed in all types of cells, including in the hematopoietic system (26). Chimeric adenovirus 5/F35 vector was effectively infected into human Mo-DCs (27), which was consistent with the findings of the present study.

Furthermore, the adenovirus vector encoded two genes simultaneously, and the two genes, CD40L and IL-2, were connected by the internal nucleic acid access site (IRES) sequence, and controlled by CMV promoter regulation, resulting in the efficient expression of the two genes. IRES is a commonly used method for producing double gene expression vectors $(28,29)$; however, the expression levels of the two genes connected via the IRES is not completely consistent, and often the gene expression level close to the promoter is high, while the gene expression level downstream of the IRES is low (30). In conclusion, the present study indicates that CD40L and IL-2 connected by IRES may be highly expressed, and IRES and IL-2 may be connected by NcoI locus (ccatgg), with the Kozak sequence (accatgg) being formed in favor of the expression of IL-2. The present study demonstrates the connection between IRES CD40L and IL-2 may lead to efficient expression. The IRES and IL-2 are connected by the $N c o$ I sites and form the Kozak Sequence (accatgg), which is conducive to increased IL-2 expression. Ad5/F35 CD40L-IL-2 is efficiently infected into human Mo-DCs and expresses CD86, CD80, CD83, CD40 and HLA-DR genes. In addition, Ad5/F35 CD40L-IL-2 stimulates the maturation of Mo-DCs and results in high levels of IL-12 production.

\section{References}

1. Cun Y, Zhang Q, Xiong C, Li M, Dai N, Zhang S and Wang D: Combined use of adenoviral vector Ad5/F35-mediated APE1 siRNA enhances the therapeutic efficacy of adenoviral-mediated p53 gene transfer in hepatoma cells in vitro and in vivo. Oncol Rep 29: 2197-2204, 2013.

2. Kim SY, Kang S, Song JJ and Kim JH: The effectiveness of the oncolytic activity induced by Ad5/F35 adenoviral vector is dependent on the cumulative cellular conditions of survival and autophagy. Int J Oncol 42: 1337-1348, 2013.
3. Hu X, Cao Y, Meng Y and Hou M: A novel modulation of structural and functional changes of mouse bone marrow derived dendritic cells (BMDCs) by interleukin-2(IL-2). Hum Vaccin Immunother 11: 516-521, 2015.

4. de Vries IJ, Lesterhuis WJ, Scharenborg NM, Engelen LP, Ruiter DJ, Gerritsen MJ, Croockewit S, Britten CM, Torensma R, Adema GJ, et al: Maturation of dendritic cells is a prerequisite for inducing immune responses in advanced melanoma patients. Clin Cancer Res 9: 5091-5100, 2003.

5. Kim R, Emi M and Tanabe K: Functional roles of immature dendritic cells in impaired immunity of solid tumour and their targeted strategies for provoking tumour immunity. Clin Exp Immunol 146: 189-196, 2006.

6. Castiello L, Sabatino M, Jin P, Clayberger C, Marincola FM, Krensky AM and Stroncek DF: Monocyte-derived DC maturation strategies and related pathways: A transcriptional view. Cancer Immunol Immunother 60: 457-466, 2011.

7. Mossoba ME and Medin JA: Cancer immunotherapy using virally transduced dendritic cells: Animal studies and human clinical trials. Expert Rev Vaccines 5: 717-732, 2006.

8. Cavallini C, Lovato O, Bertolaso A, Pacelli L, Zoratti E, Zanolin E, Krampera M,Zamò A, Tecchio C, Cassatella MA, et al: The TNF-family cytokine TL1A inhibits proliferation of human activated B cells. PLoS One 8: e60136, 2013.

9. Erdmann M and Schuler-Thurner B: Towards a standardized protocol for the generation of monocyte-derived dendritic cell vaccines. Methods Mol Biol 595: 149-163, 2010.

10. Guo Z, Liu H, He XP, Tan XH, Zhou Y, Chen X, Shi YJ, Liu XD and Chen HR: A clinical study of cytokine-induced killer cells for the treatment of refractory lymphoma. Oncol Lett 2: 531-536, 2011.

11. Boudreau JE, Bonehill A, Thielemans K and Wan Y: Engineering dendritic cells to enhance cancer immunotherapy. Mol Ther 19: 841-853, 2011.

12. Morelli AE, Zahorchak AF, Larregina AT, Colvin BL, Logar AJ, Takayama T, Falo LD and Thomson AW: Cytokine production by mouse myeloid dendritic cells in relation to differentiation and terminal maturation induced by lipopolysaccharide or CD40 ligation. Blood 98: 1512-1523, 2001.

13. Kelleher M and Beverley PC: Lipopolysaccharide modulation of dendritic cells is insufficient to mature dendritic cells to generate CTLs from naive polyclonal CD $8^{+} \mathrm{T}$ cells in vitro, whereas CD40 ligation is essential. J Immunol 167: 6247-6255, 2001.

14. Tcherepanova IY, Adams MD, Feng X, Hinohara A, Horvatinovich J, Calderhead D, Healey D and Nicolette CA: Ectopic expression of a truncated CD40L protein from synthetic post-transcriptionally capped RNA in dendritic cells induces high levels of IL-12 secretion. BMC Mol Biol 9: 90, 2008.

15. Hernandez MG, Shen L and Rock KL: CD40-CD40 ligand interaction between dendritic cells and $\mathrm{CD} 8^{+} \mathrm{T}$ cells is needed to stimulate maximal $\mathrm{T}$ cell responses in the absence of $\mathrm{CD} 4^{+} \mathrm{T}$ cell help. J Immunol 178: 2844-2852, 2007.

16. Thacker EE, Nakayama M, Smith BF, Bird RC, Muminova Z, Strong TV,TimaresL,Korokhov N,O'Neill AM,deGruijl TD, etal: A genetically engineered adenovirus vector targeted to CD40 mediates transduction of canine dendritic cells and promotes antigen-specific immune responses in vivo. Vaccine 27: 7116-7124, 2009.

17. Kikuchi T, Moore MA and Crystal RG: Dendritic cells modified to express CD40 ligand elicit therapeutic immunity against preexisting murine tumors. Blood 96: 91-99, 2000.

18. Tada Y, O-Wang J, Yu L, Shimozato O, Wang YQ, Takiguchi Y, Tatsumi K, Kuriyama T, Takenaga K, Sakiyama S, et al: T-cell-dependent antitumor effects produced by CD40 ligand expressed on mouse lung carcinoma cells are linked with the maturation of dendritic cells and secretion of a variety of cytokines. Cancer Gene Ther 10: 451-456, 2003.

19. Wolf B, Schwarzer A, Côté AL, Hampton TH, Schwaab T, Huarte E, Tomlinson CR, Gui J, Fisher JL, Fadul CE, et al: Gene expression profile of peripheral blood lymphocytes from renal cell carcinoma patients treated with IL-2, interferon- $\alpha$ and dendritic cell vaccine. PLoS One 7: e50221, 2012.

20. Stax AM, Crul C, Kamerling SW, Schlagwein N, van der Geest RN, Woltman AM and van Kooten C: CD40L stimulation of rat dendritic cells specifically favors the IL-12/IL-10 ratio resulting in a strong T cell stimulatory capacity. Mol Immunol 45: 2641-2650, 2008.

21. Nagy ZS, Ross JA, Rodriguez G, Balint BL, Szeles L, Nagy L and Kirken RA: Genome wide mapping reveals PDE4B as an IL-2 induced STAT5 target gene in activated human PBMCs and lymphoid cancer cells. PLoS One 8: e57326, 2013. 
22. Gonzalez-Carmona MA, Lukacs-Kornek V, Timmerman A Shabani S, Kornek M, Vogt A, Yildiz Y, Sievers E, Schmidt-Wolf IG, Caselmann WH, et al: CD40ligand-expressing dendritic cells induce regression of hepatocellular carcinoma by activating innate and acquired immunity in vivo. Hepatology 48 157-168, 2008.

23. DeBenedette MA, Calderhead DM, Tcherepanova IY, Nicolette CA and Healey DG: Potency of mature CD40L RNA electroporated dendritic cells correlates with IL-12 secretion by tracking multifunctional CD $8(+) / C D 28(+)$ cytotoxic T-cell responses in vitro. J Immunother 34: 45-57, 2011.

24. Rousseau RF, Biagi E, Dutour A, Yvon ES, Brown MP, Lin T, Mei Z, Grilley B, Popek E, Heslop HE, et al: Immunotherapy of high-risk acute leukemia with a recipient (autologous) vaccine expressing transgenic human CD40L and IL-2 after chemotherapy and allogeneic stem cell transplantation. Blood 107: 1332-1341, 2006

25. Balamotis MA, Huang K and Mitani K: Efficient delivery and stable gene expression in a hematopoietic cell line using a chimeric serotype 35 fiber pseudotyped helper-dependent adenoviral vector. Virology 324: 229-237, 2004.

26. Gaggar A, Shayakhmetov DM and Lieber A: CD46 is a cellular receptor for group B adenoviruses. Nat Med 9: 1408-1412, 2003.
27. Ophorst OJ, Kostense S, Goudsmit J, De Swart RL, Verhaagh S, Zakhartchouk A, Van Meijer M, Sprangers M, Van Amerongen G, Yüksel S, et al: An adenoviral type 5 vector carrying a type 35 fiber as a vaccine vehicle: DC targeting, cross neutralization, and immunogenicity. Vaccine 22: 3035-3044, 2004.

28. Vitoriano-Souza J, Moreira N, Teixeira-Carvalho A, Carneiro CM, Siqueira FA, Vieira PM, Giunchetti RC, Moura SA, Fujiwara RT, Melo MN, et al: Cell recruitment and cytokines in skin mice sensitized with the vaccine adjuvants: Saponin, incomplete Freund's adjuvant, and monophosphoryl lipid A. PLoS One 7: e40745, 2012.

29. Yang S, Cohen CJ, Peng PD, Zhao Y, Cassard L, Yu Z, Zheng Z, Jones S, Restifo NP, Rosenberg SA, et al: Development of optimal bicistronic lentiviral vectors facilitates high-level TCR gene expression and robust tumor cell recognition. Gene Ther 15: 1411-1423, 2008

30. Ngoi SM, Chien AC and Lee CG: Exploiting internal ribosome entry sites in gene therapy vector design. Curr Gene Ther 4: 15-31, 2004. 